\title{
Cognate HLA absence in trans diminishes human NK cell education
}

\author{
Vanessa Landtwing, ${ }^{1}$ Ana Raykova, ${ }^{1}$ Gaetana Pezzino, ${ }^{2}$ Vivien Béziat, ${ }^{3}$ Emanuela Marcenaro, ${ }^{4,5}$ Claudine Graf, ${ }^{6}$ \\ Alessandro Moretta, ${ }^{4,5}$ Riccarda Capaul, ${ }^{7}$ Andrea Zbinden, ${ }^{7}$ Guido Ferlazzo, ${ }^{2}$ Karl-Johan Malmberg, ${ }^{8,9,10}$ \\ Obinna Chijioke, ${ }^{1,1}$ and Christian Münz ${ }^{1}$
}

\begin{abstract}
'Viral Immunobiology, Institute of Experimental Immunology, University of Zürich, Zürich, Switzerland. 2Laboratory of Immunology and Biotherapy, University of Messina and Cell Therapy Program Azienda Ospedaliera Universitaria Policlinico G. Martino, Messina, Italy. ${ }^{3}$ Laboratory of Human Genetics of Infectious Diseases, Necker Branch, INSERM U1163, Paris, France. ${ }^{4}$ Department of Experimental Medicine, and ${ }^{5}$ Center of Excellence for Biomedical Research, University of Cenoa, Genova, Italy. ${ }^{6} 3 \mathrm{rd}$ Medical Department and Center for Thrombosis and Hemostasis, University Medical Center of the Johannes Gutenberg University, Mainz, Germany. IInstitute of Medical Virology, University of Zürich, Zürich, Switzerland. ${ }^{8}$ Center for Infectious Medicine, Department of Medicine, Karolinska Institutet, Huddinge, Sweden. ${ }^{9}$ Institute for Cancer Research, Oslo University Hospital, Oslo, Norway. ${ }^{10 T h e ~ K G ~ J e b s e n ~ C e n t e r ~ f o r ~ C a n c e r ~ I m m u n o t h e r a p y ~ I n s t i t u t e ~ o f ~ C l i n i c a l ~ M e d i c i n e, ~}$
\end{abstract}

University of Oslo, Oslo, Norway. "Institute of Surgical Pathology, University Hospital Zürich, Zürich, Switzerland.

\begin{abstract}
NK cells are innate lymphocytes with protective functions against viral infections and tumor formation. Human NK cells carry inhibitory killer cell Ig-like receptors (KIRs), which recognize distinct HLAs. NK cells with KIRs for self-HLA molecules acquire superior cytotoxicity against $\mathrm{HLA}^{-}$tumor cells during education for improved missing-self recognition. Here, we reconstituted mice with human hematopoietic cells from donors with homozygous KIR ligands or with a mix of hematopoietic cells from these homozygous donors, allowing assessment of the resulting KIR repertoire and NK cell education. We found that coreconstitution with 2 KIR ligand-mismatched compartments did not alter the frequency of KIR-expressing NK cells. However, NK cell education was diminished in mice reconstituted with parallel HLA compartments due to a lack of cognate HLA molecules on leukocytes for the corresponding KIRs. This change in NK cell education in mixed human donor-reconstituted mice improved NK cell-mediated immune control of EBV infection, indicating that mixed hematopoietic cell populations could be exploited to improve NK cell reactivity against leukotropic pathogens. Taken together, these findings indicate that leukocytes lacking cognate HLA ligands can disarm KIR+ NK cells in a manner that may decrease HLA' tumor cell recognition but allows for improved NK cell-mediated immune control of a human $\gamma$-herpesvirus.
\end{abstract}

\section{Introduction}

NK cells are prototypic innate lymphocytes and have originally been identified by their ability to spontaneously kill transformed and infected cells (1-3). They recognize their targets by balancing signals of activating and inhibitory receptors, resulting in missing-self recognition upon loss of inhibitory ligands, mostly MHC class I molecules, and altered-self recognition upon gain of activating ligands on the surface of encountered cells (4-6). The ability of NK cells to detect too few inhibitory ligands or too many activating ligands in reference to unaltered host tissue is thought to be acquired by NK cells in a continuous process called "education" or "licensing" via the interaction of inhibitory NK cell receptors and MHC class I molecules (7-9). Whether NK cell education is mediated "in cis" (on the NK cell itself) or "in trans" (by neighboring cells) or both is still a matter of debate (10-13), however, the hematopoietic compartment seems to be sufficient in both mice and humans for NK cell education $(11,14)$. Along these lines, the recipient HLA haplotype was not found to significantly abolish donor NK cell alloreactivity via education during the first 3 months after

Authorship note: $\mathrm{O}$. Chijioke and C. Münz contributed equally to this work.

Conflict of interest: The authors have declared that no conflict of interest exists. Submitted: February 8, 2016; Accepted: July 21, 2016.

Reference information: J Clin Invest. 2016;126(10):3772-3782. doi:10.1172/JCI86923.
BM transplantation (15). Within the hematopoietic compartment, multiple cell populations probably contribute to NK cell education, including the partial contribution of $\mathrm{T}$ cells that was previously identified in the mouse (12). The process of education safeguards NK cell cytotoxicity by either "arming" cells that express, in parallel to activating NK cell receptors, inhibitory receptors that are sufficiently engaged by unaltered host cells, or by "disarming" through continuous engagement of activating receptors without inhibitory signals $(16,17)$. While most activating NK cell receptors are nearly uniformly expressed on all mature NK cells, inhibitory receptors are expressed on subsets, whose contribution to the NK cell repertoire of an individual is guided by genetic and environmental factors, e.g., the genomic repertoire of killer Ig-like receptors (KIRs) and persistent CMV (HCMV) infection in humans (18). This composition of the NK cell repertoire becomes particularly important during primary infection with pathogens like herpesviruses, which clinically identify individuals with primary immunodeficiencies that impair NK cell functions (19).

One of these is the infection with the human oncogenic $\gamma$-herpesvirus EBV. EBV persistently infects more than $90 \%$ of the human adult population (20). While primary infection is usually asymptomatic in children, adolescents more frequently suffer from infectious mononucleosis (IM) during their first encounter with $\operatorname{EBV}(21,22)$. While EBV is associated with B cell and epithe- 
A
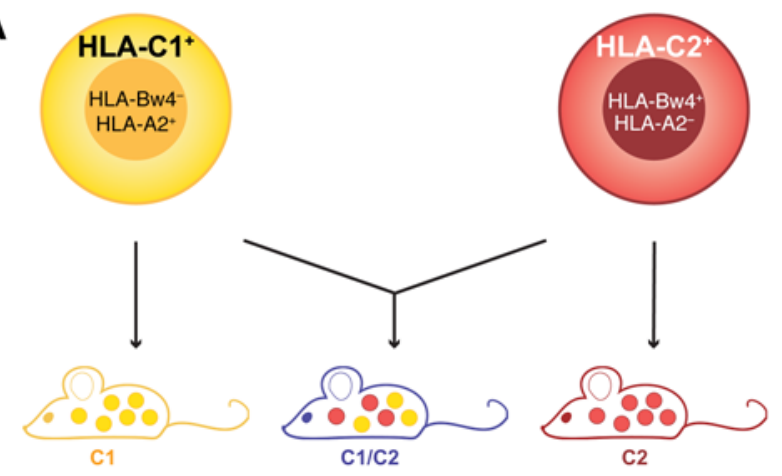

B
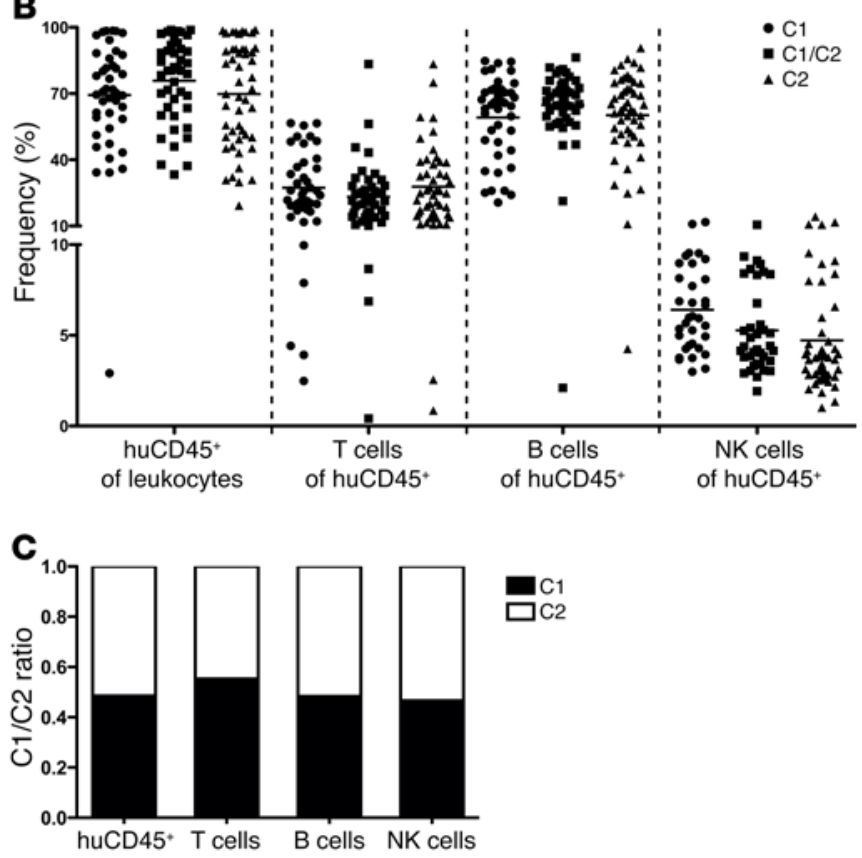

lial malignancies through its viral oncogenes that are expressed during latent infection, lytic replication that produces infectious virions seems to be poorly controlled during IM and causes the characteristic immune pathogenic $\mathrm{CD} 8^{+} \mathrm{T}$ cell lymphocytosis of this disease (23). NK cells were reported to expand during IM (21, $24,25)$ and seem to particularly restrict lytic EBV infection that is deregulated during $\operatorname{IM}(25,26)$. Interestingly, and in contrast to the prototypic terminally differentiated NK cell-expanding pathogen HCMV, EBV seems to drive the proliferation of early differentiated non-KIR-educated NK cells $(25,27)$. These NK cells have been reported to recognize $\mathrm{B}$ cells that lytically replicate $\mathrm{EBV}$ via recognition by the activating NK cell receptors NKG2D and DNAM-1 (28), and the respective ligands for these receptors seem to be upregulated during transition from latent to lytic EBV infection $(25,28)$. Loss of NK cells leads to elevated viral loads of WT, but not lytic replication-incompetent, EBV in vivo (26). This in turn causes hallmarks of IM, including enhanced $\mathrm{CD}^{+} \mathrm{T}$ cell expansion and lymphadenopathy, as well as weight loss and EBVassociated tumorigenesis in EBV-infected experimental animals (26). Moreover, the protective early differentiated NK cell subset decreases in frequency within the NK cell repertoire of humans during the first decade of life (25) and therefore could explain the
Figure 1. Mixed reconstitution of human immune system compartments from HLA-mismatched HPCs in NSG mice. (A) Representative experimental overview. Three types of experimental groups were used: two groups reconstituted homozygously for $\mathrm{HLA}-\mathrm{C}$ and -B allotypes (HLA-C1, - C2, and -Bw4), while disparate for HLA-A2, and the third group with a mix of both. (B) Reconstitution of human immune cell compartments in the 3 experimental groups as a percentage of human CD45+ lymphocytes. (C) Ratio of HLA-C1 donor versus HLA-C2 donor frequencies as distinguished by HLA-A2 expression in immune cell compartments of mixed reconstituted huNSC mice. Data were pooled from at least 4 independent experiments. $n=34-49$. Bars represent the mean in the respective graphs.

increased susceptibility to IM in adolescents. Thus, EBV infection seems to be sensitive to the balance between non-KIR-educated NK cells, which are either KIR- or whose KIRs have not encountered cognate MHC class I molecules, and KIR-educated NK cells.

In our study, we investigated NK cell repertoire generation and NK cell education and their influence on the control of EBV infection in mice with reconstituted human immune system components (huNSG mice). For this purpose, we either reconstituted human NK cell compartments from hematopoietic progenitor cells (HPCs) that homozygously carry MHC class I molecules for distinct KIRs individually or in mixed chimeras. We found that the distribution of KIRs on the reconstituted human NK cells is donor autonomous and independent of environmental MHC class I molecules, while education, mediated by the hematopoietic compartment in this setting, is compromised as soon as a subset of immune cells does not carry the cognate MHC class I molecules. Thus, abrogation of the educational process seems to result from activating receptor engagement with co-reconstituting hematopoietic cells in the absence of inhibitory signaling. This results in higher frequencies of noneducated NK cells in the mixed reconstitutions and an improved immune control of EBV infection. Therefore, our findings are most consistent with a disarming model of KIR-mediated education, resulting in NK cell compartments, which, because of the inflammatory environment of EBV infection, nevertheless efficiently control this human tumor virus.

\section{Results}

An in vivo model with parallel reconstitution of 2 donors' immune compartments in NSG mice. Double-unit umbilical cord blood transplantation is successfully being used to improve engraftment and decrease mortality in patients $(29,30)$. During the engraftment process, mostly 1 donor prevails, which leads to single-unit engraftment $(31,32)$. Human NK cell education, however, has been shown to be driven by the hematopoietic compartment (14). Therefore, little data are available on how the absence of cognate human MHC class I molecules, namely cognate HLA class I molecules, on hematopoietic cells influences the education and KIR repertoire of human NK cells in trans. In order to study the effect of cognate versus noncognate HLA on NK cell development, we reconstituted NOD-SCID $\gamma_{\mathrm{c}}{ }^{-/-}$(NSG) mice with HLA-typed human HPCs isolated from fetal livers (huNSG mice). Human fetal liver (HFL) donors were chosen to be homozygous for the HLA-C and -B allotypes (HLA-C1, -C2, and -Bw4), which are recognized by KIR2DL and KIR3DL molecules, respectively. Furthermore, donors were disparate for HLA-A2 expression, which allowed us to track the progeny of specific donors in mixed reconstitution 
A $\%$ of KIR subsets in single (C1)
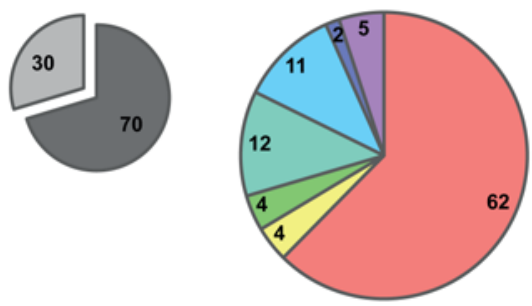

C $\%$ of KIR subsets in mixed (C1)
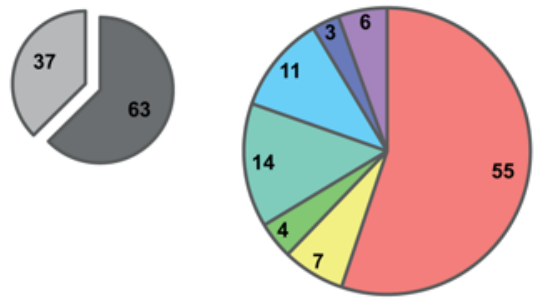

E $\%$ of KIR subsets in HLA-C1 HFL
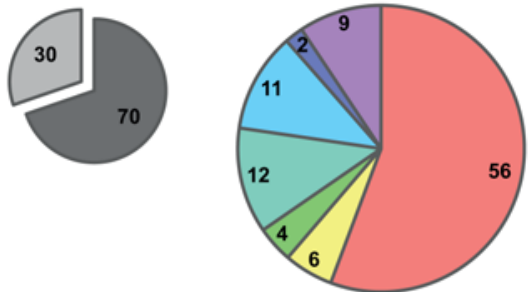

B
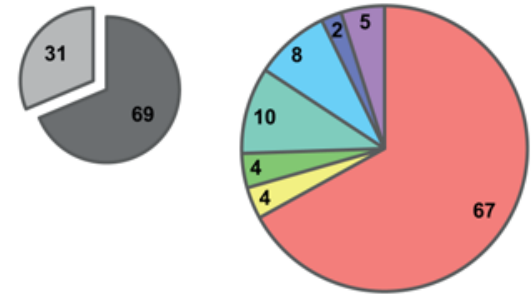

D $\%$ of KIR subsets in mixed (C2)
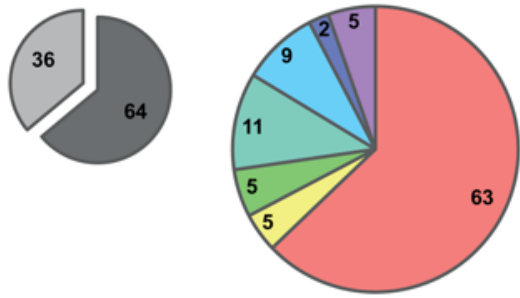

F \% of KIR subsets in HLA-C2 HFL

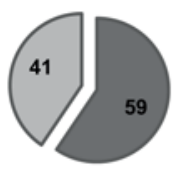

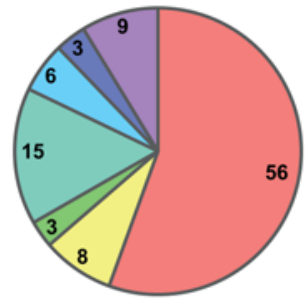

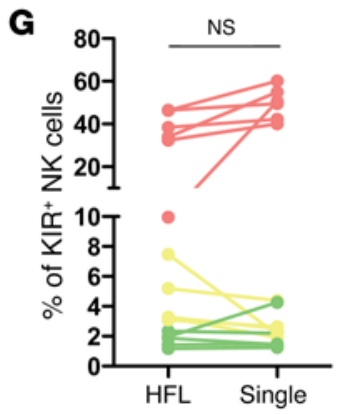

H

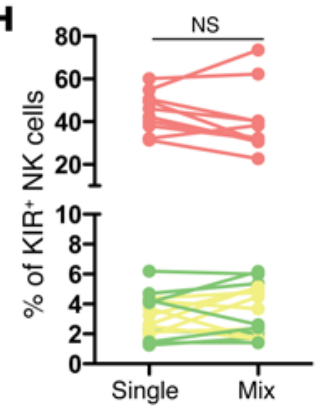

Figure 2. HLA haplotype in trans does not influence the KIR repertoire in steady state. HuNSC mice were single reconstituted with donors homozygous for HLA-C and -B allotypes (HLA-C1, - $C 2$, and -Bw4) (single) or reconstituted with 2 donors disparate for allotype and HLA-A2 expression (mixed). Gray pie charts depict the frequencies of $\mathrm{KIR}^{+}$and $\mathrm{KIR}^{-}$NK cells, and colored pie charts represent the indicated KIR+ NK cell subsets. (A-F) Representation of KIR-expressing NK cell subsets as a percentages of total KIR+ NK cells in liver of huNSG mice (A-D) or of HFL donors (E and F). KIR subsets of liver NK cells from huNSG mice reconstituted with single donors homozygous for HLA-C1 (A) or -C2 (B). (C and D) KIR subsets of liver NK cells from mixed reconstituted huNSG mice, with NK cells derived from HLA-C1 (C) and HLA-C2 (D) donors. (E and F) KIR subsets of NK cells from HLA-C1 (E) or -C2 (F) HFLs. $n=3$. ( $(\mathbf{G}$ and $\mathbf{H}$ ) Mean percentage per experiment of single KIR+ liver NK cells as derived from total NK cells of huNSG mice. Frequencies of $\mathrm{KIR}^{+}$NK cells in single reconstituted huNSG mice as compared with those in HFL donors (G) and mixed reconstituted huNSG mice (H). Data were pooled data from at least 3 independent experiments. $n=11-14$ mice per group. ( $\mathbf{C}$ and $\mathbf{H}$ ) Dots represent the mean of the cell population in separate experiments (paired 2-tailed Student's $t$ test).

experiments (Figure 1A). Three types of experimental mouse groups were reconstituted in this study. Two single-donor hematopoietic cell-reconstituted groups (referred to hereafter as single reconstituted) homozygous for either HLA-C1 (recognized by KIR2DL2/3) or HLA-C2 (recognized by KIR2DL1) and HLA-Bw4 (recognized by KIR3DL1) were set up in parallel to a third group that was reconstituted with a mix of hematopoietic cells from both donors (referred to hereafter as mixed reconstituted). The results of reconstitution of human $\mathrm{CD}_{4} 5^{+}$leukocytes in general, as well as the frequencies of reconstituted T cells, B cells, and NK cells, were comparable to those of previous studies conducted in our laboratory (Figure 1B) $(33,34)$. Notably, mixed reconstituted huNSG mice developed cells from both donors side by side in all the reconstituted human immune system compartments (Figure 1C). Thus, we established a model to study the influence of cog- nate HLA presence or absence in trans on NK cell education and KIR repertoire development.

Development of the KIR repertoire on NK cells is not influenced by the HLA haplotype in trans. Next, we analyzed the KIR repertoire of NK cells in liver and spleen of reconstituted huNSG mice and compared it with the KIR repertoire as present in the fetal liver of the original donor (Figure 2, Supplemental Figure 1 for gating, Supplemental Figure 2, and data not shown; supplemental material available online with this article; doi:10.1172/JCI86923DS1). In order to detect differences in mixed reconstituted huNSG mice, NK cells from these mice were separately evaluated according to donor origin. The overall diversity of the KIR repertoire was comparable to that of all groups as well as to that in the donor HFLs, and no preferential expansion of KIR subsets could be seen (Figure 2, A-F). When relating the KIR frequencies of reconstituted 
A $\%$ of KIR subsets in single (C1)
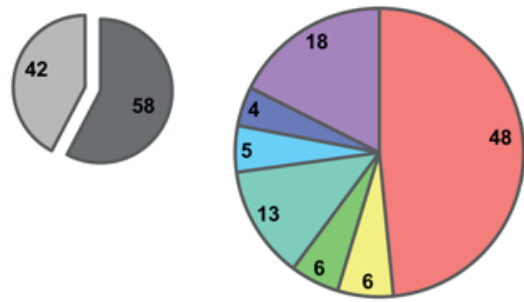

B $\%$ of KIR subsets in mixed (C1)
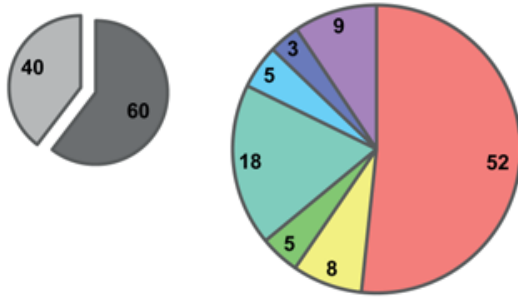

C $\%$ of KIR subsets in mixed (C2)
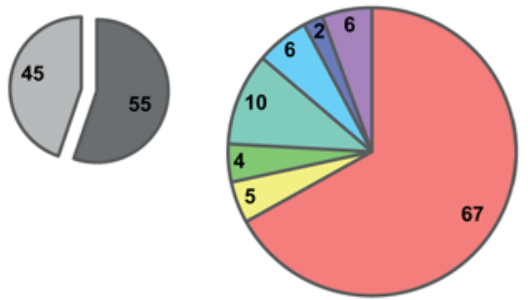

D $\%$ of $\mathrm{KIR}$ subsets in single (C2)

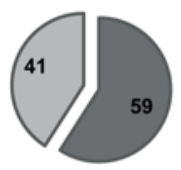

$\square \mathrm{KIR}^{+}$

$\square \mathrm{KIR}^{-}$

KIR2DL1+

$\square \mathrm{KIR} 2 \mathrm{DL} 2 / 3$

$\square$ KIR3DL1+

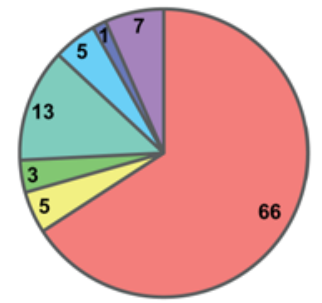

$\square \mathrm{KIR} 2 \mathrm{DL} 1+\mathrm{KIR} 2 \mathrm{DL} 2 / 3^{+}$

KIR2DL1+KIR3DL1+

KIR2DL2/3+KIR3DL1

KIR2DL1+KIR2DL2/3+KIR3DL1+
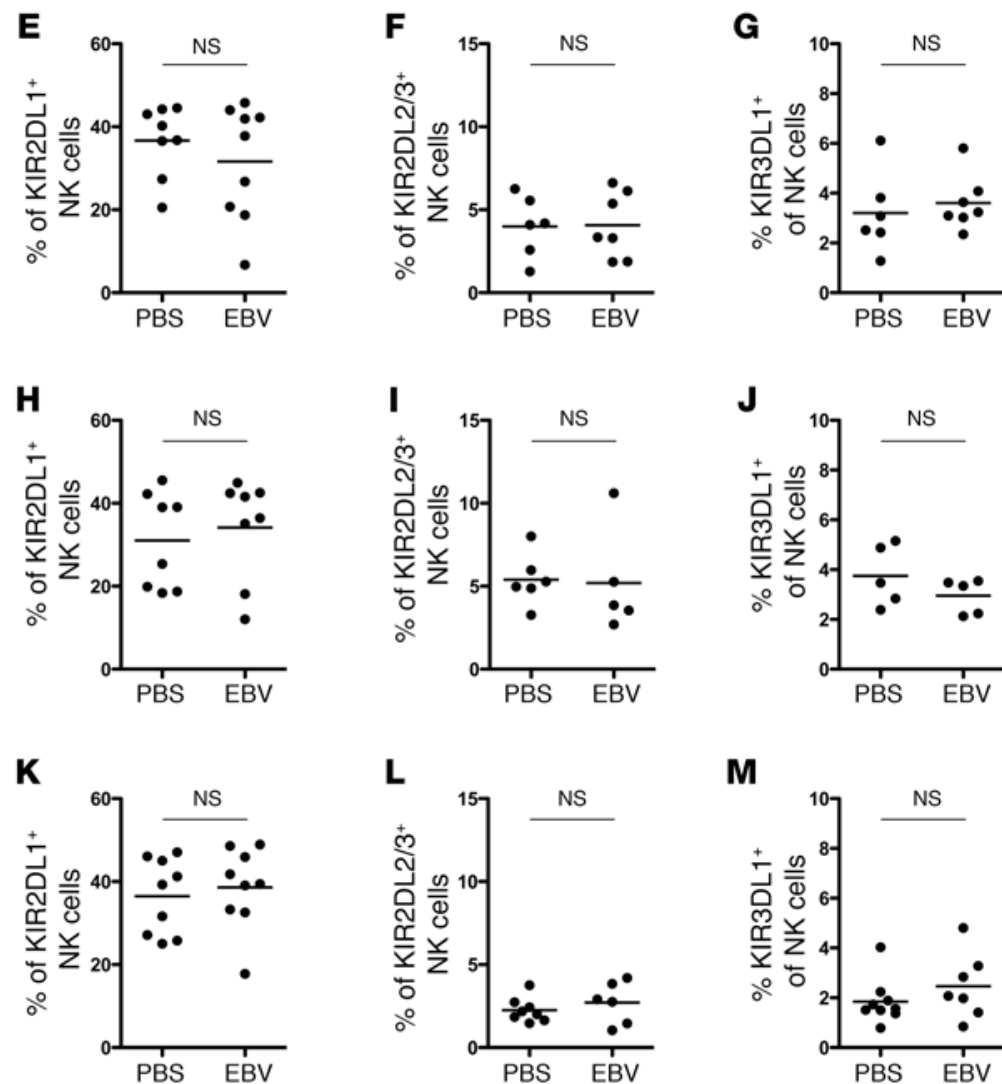

M

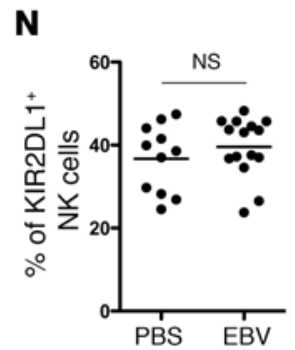

O

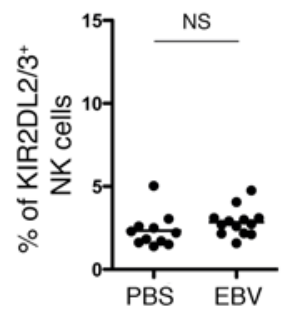

$\mathbf{P}$

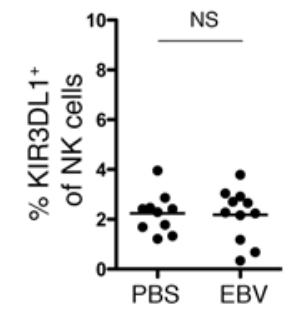

Figure 3. Infection with EBV does not lead to skewing of KIR repertoire. HuNSG mice were single reconstituted with donors homozygous for HLA-C and -B allotypes (HLA-C1, - C2, and -Bw4) or reconstituted with 2 donors disparate for allotype and HLA-A2 expression (mixed). Gray pie charts depict the frequencies of KIR+ and KIR- NK cells, and colored pie charts represent the indicated KIR+ NK cell subsets. (A-D) Subsets of KIR-expressing NK cells as a percentages of total KIR+ NK cells in spleens of EBV-infected huNSG mice. (E-P) Frequency of single KIR+ NK cells in spleens of huNSG mice at endpoint. Comparison of PBS controls with EBV-infected animals for frequency of KIR2DL1+, KIR2DL2/3+, or KIR3DL1+ NK cells in homozygous reconstituted mice with HLA-C1 donor (E-C), mixed reconstituted mice with cells derived from HLA-C1 (H-J) or - C2 (K-M) donors, and homozygous reconstituted mice with cells from HLA-C2 donors (M-P). Data are pooled from at least 3 independent experiments. $n=8-15$ mice per group. Bars represent the mean (unpaired Student's $t$ test).

mice to their specific HFL donors, a correlation could be detected between the two (Figure $2 \mathrm{G}$ and Supplemental Figure 2). Namely, HFL donors with, for example, high frequencies of KIR2DL1, KIR2DL2/3, or KIR3DL1 single-positive NK cells reconstituted the respective NK cell subsets also at higher frequencies. Importantly, in mixed reconstituted huNSG mice, the presence of noncognate HLA in trans did not significantly change the KIR repertoire (Figure 2, C and D), and no differences were detectable when comparing specific KIR frequencies with those of single reconstituted mice
(Figure 2H and Supplemental Figure 2). In spleen, a KIR repertoire composition similar to that in the liver was observed but could not be compared with the splenic NK cell repertoire of the HFL donors (data not shown). Hence, it seems that the absence of cognate HLA on co-reconstituting hematopoietic cells does not influence the development of the KIR repertoire in the steady state.

$E B V$ infection does not force skewing of the KIR repertoire on NK cells in huNSG mice. Previous work has established profound effects of human CMV infection on the NK cell KIR repertoire and 
A
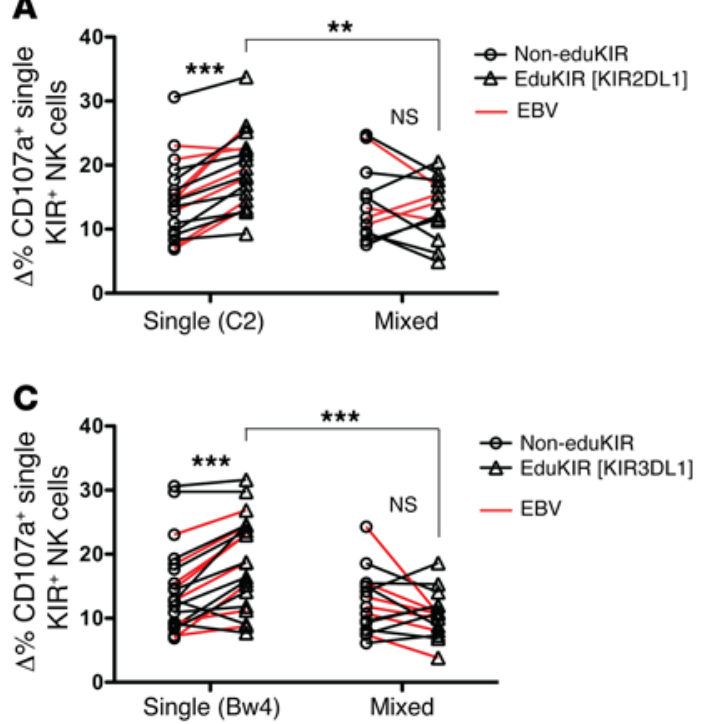

B
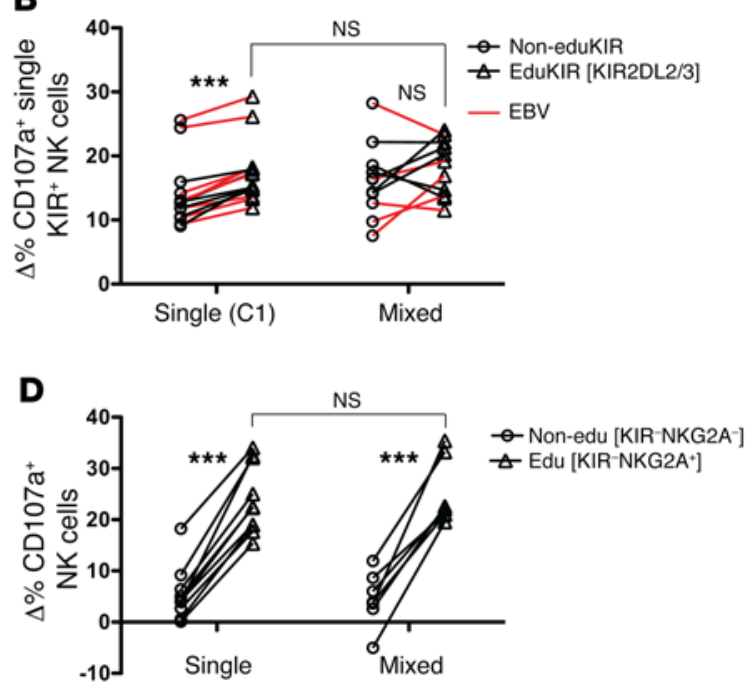

Figure 4. Loss of NK cell education in the presence of noncognate MHC class I in mixed reconstituted huNSG mice. huNSG mice were single reconstituted with donors homozygous for HLA-C and -B allotypes (HLA-C1, -C2, and -Bw4) (Single) or reconstituted with 2 donors disparate for allotype and HLA-A2 expression (Mixed). $\triangle \%$ CD107a refers to the difference in degranulation with and without K562 restimulation. The average \pm SEM of spontaneous degranulation was $4.04 \% \pm 0.79 \%$. (A-C) Degranulation of single KIR+ NK cells after incubation of K562 cells with splenocytes from huNSG mice reconstituted with (A) homozygous HLA-C2 or mixed donors ( $n=12-18$ mice/group), (B) homozygous HLA-C1 or mixed donors ( $n=11-14$ mice/group), or (C) homozygous HLA-Bw4 or mixed donors ( $n=15-20$ mice/group), infected (red lines) or not (black lines) with EBV for 5 weeks. According to single reconstituted allotypes, eduKIR denotes educated NK cells and non-eduKIR denotes noneducated NK cells gated on cells derived from donors used for single reconstitution in both single and mixed reconstituted huNSG mice, respectively. (D) Degranulation of KIR-NKG2A- (non-edu) and KIR-NKG2A+ (edu) NK cells after incubation of $\mathrm{K} 562$ cells with splenocytes from single or double (Mixed) reconstituted huNSC mice ( $n=7-10 /$ group), gated on cells derived from donors used for single reconstitution. Data in $\mathbf{A}-\mathbf{C}$ were pooled from at least 3 independent experiments, data in $\mathbf{D}$ are compound data from 2 independent experiments. ${ }^{* *} P<0.01$ and ${ }^{* * *} P<0.001$, by multiple paired Student's $t$ tests.

expansion of KIR educated NKG2C $\mathrm{C}^{+} \mathrm{NK}$ cells $(18,35)$. In an effort to examine whether the KIR repertoire could be skewed by another herpesvirus, huNSG mice were infected with EBV and monitored for 5 weeks. At the endpoint, the livers and spleens of infected animals were analyzed for KIR expression in NK cells (Figure 3 and Supplemental Figure 3). EBV infection did not lead to obvious changes in the $\mathrm{KIR}^{+} \mathrm{NK}$ cell subset composition in single or mixed reconstituted huNSG mice (Figures 3, A-D). Furthermore, when comparing frequencies of NK cells that were single positive for KIR2DL1, KIR2DL2/3, or KIR3DL1 in EBV-infected animals with frequencies in controls, no differences could be detected (Figures 3, E-P). Stable KIR repertoires were also observed in the livers of EBV-infected huNSG mice (Supplemental Figure 3). It therefore appears that EBV infection is not able to induce skewing of the KIR repertoire in huNSG mice.

Absence of cognate HLA abrogates NK cell education in huNSG mice. Whether human NK cells in mouse models reconstituted with human immune system components are educated has not been addressed so far. To quantify education in huNSG mice, we assessed the degranulation of single $\mathrm{KIR}^{+}$splenic NK cells toward a prototypic NK cell target, MHC class I-negative erythroleukemic K562 cells, in the context of the educating HLA ligands. The presence of solely the educating, cognate HLA, as in the case of single reconstituted huNSG mice, resulted in education of KIR2DL1 ${ }^{+}$, KIR2DL2 $/ 3^{+}$, and KIR3DL1 ${ }^{+} \mathrm{NK}$ cells in HLA-C2, -C1, and -Bw4 reconstituted animals, respectively (Figures $4, \mathrm{~A}-\mathrm{C}$ ), with functional differences similar to those between educated and noneducat- ed NK cells from healthy volunteers $(36,37)$. Furthermore, acute infection with EBV did not influence this process. In contrast, however, the additional presence of noncognate HLA in trans in mixed reconstituted huNSG mice prevented NK cells from being educated (Figures 4, A-C). Interestingly, education was lost regardless of the donor ratio in the mixed reconstituted animals and was even observed when the cognate HLA- leukocyte reconstitution only amounted to $5 \%$ of the total human immune components (Supplemental Figure $4, \mathrm{~A}-\mathrm{C}$ ). This argues against a dose effect of cognate HLA and for the disarming model in KIR-mediated NK cell education as an active process, whereby activating receptor engagement in the absence of inhibitory signaling renders NK cells hyporesponsive. When looking at education in the context of NKG2A and its ubiquitously present educating HLA-E ligand, both mixed and single reconstituted huNSG mice showed equal education, consistent with invariant HLA-E expression by all HFL donors (Figure 4D). Additionally, we could also show that education was detectable in the livers of huNSG mice, albeit at decreased overall degranulation capacity, consistent with the findings of previous studies $(38,39)$. Therefore, human NK cell education takes place in huNSG mice, independently of EBV infection, is mediated by the hematopoietic compartment, and is reliant on the presence of cognate HLA on all hematopoietic cells. Furthermore, the abrogation of NK cell education in mixed reconstituted animals seems to be dependent on the loss of inhibitory signaling in trans.

Noneducated NK cells still display inhibition in response to cognate $H L A$. In addition to analyzing the education of human NK cells in 
A
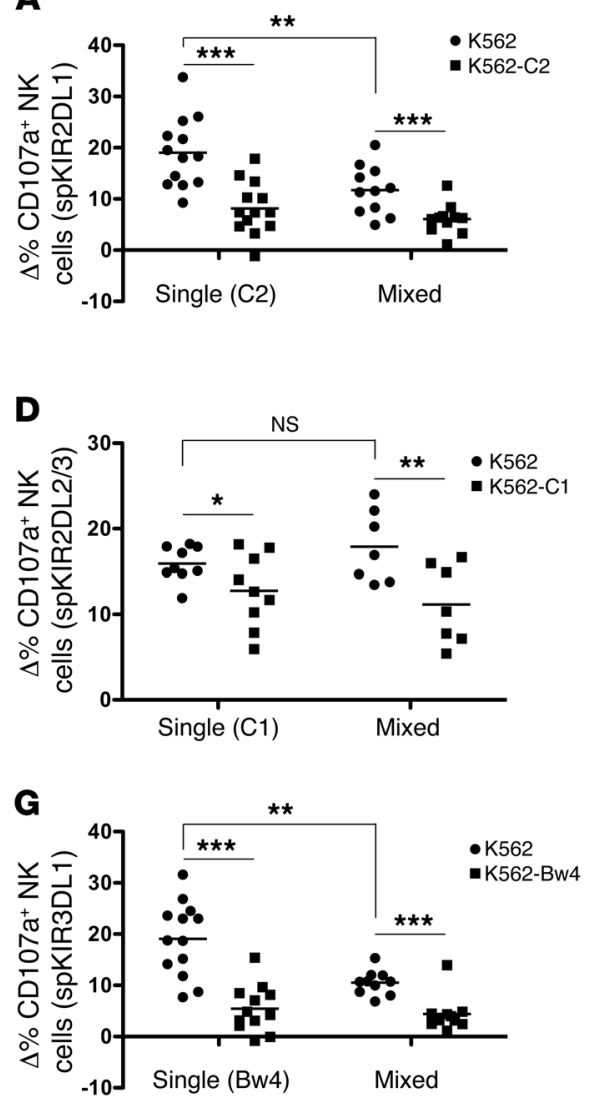

B

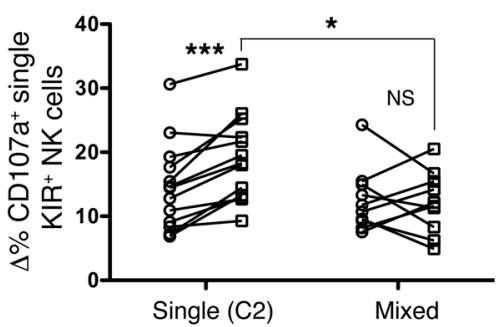

E
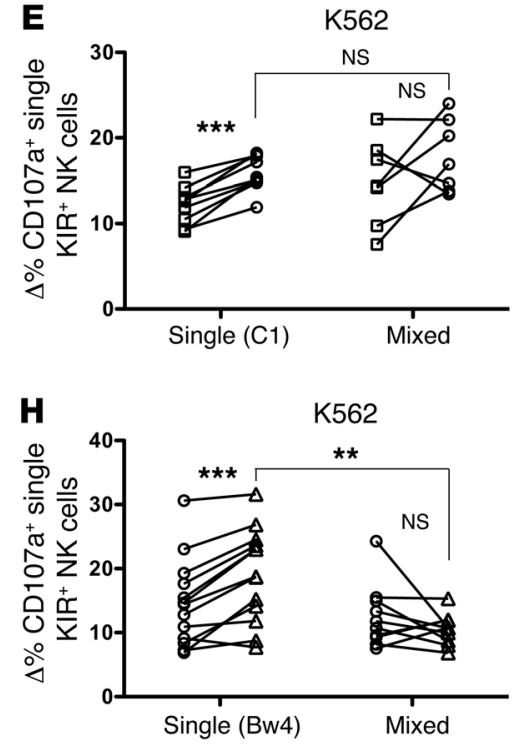

C

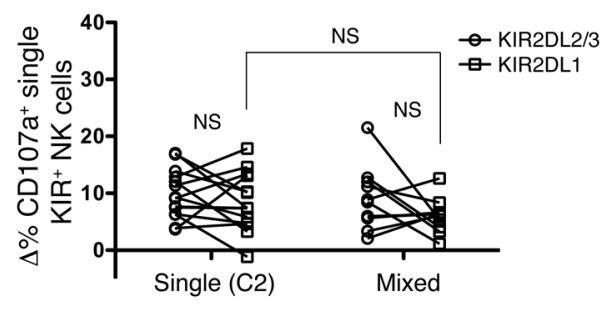

$\mathbf{F}$

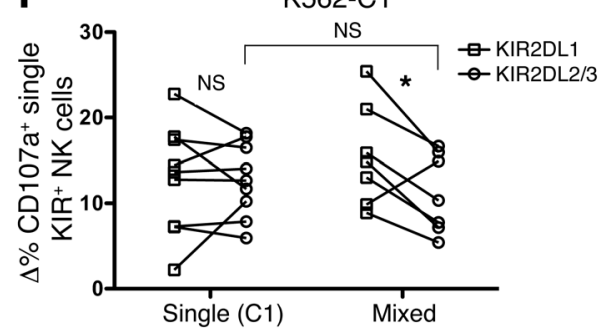

I K562-Bw4

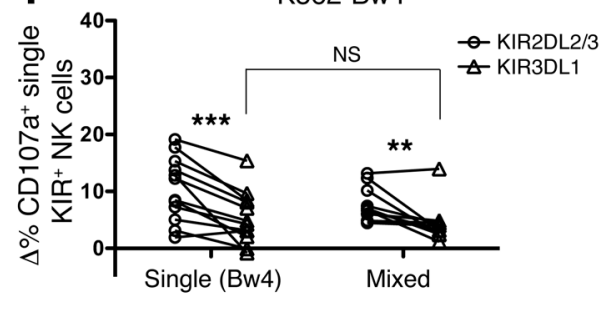

Figure 5. Inhibition by cognate MHC class I ligands is maintained in noneducated NK cells. huNSC mice were single reconstituted with donors homozygous for HLA-C and -B allotypes (HLA-C1, -C2, and -Bw4) (Single) or reconstituted with 2 donors disparate for allotype and HLA-A2 expression (Mixed) and incubated with MHC class I-negative target cells (K562) or K562 transfectants expressing HLA-C1, -C2, or -Bw4 MHC class I ligands. $\triangle \%$ CD107a refers to the difference in degranulation with and without K562 restimulation. Average \pm SEM of spontaneous degranulation was 3.55\% $\pm 0.73 \%$. (A-C) Degranulation of single KIR2DL1+ NK cells after incubation of K562 or K562-C2 cells with splenocytes from huNSG mice reconstituted with HLA-C2 homozygous or mixed donors (A) and paired analysis for incubation with K562 cells (B) or K562-C2 transfectants (C). (D-F) Degranulation of single KIR2DL2/3+ NK cells after incubation of K562 or K562-C1 cells with splenocytes from huNSG mice reconstituted with HLA-C1 homozygous or mixed donors (D) and paired analysis for incubation with K562 cells (E) or K562-C1 transfectants (F). (G-I) Degranulation of single KIR3DL1+ NK cells after incubation of K562 or K562-Bw4 cells with splenocytes from huNSG mice reconstituted with HLA-Bw4 homozygous or mixed donors (G) and paired analysis for incubation with K562 cells (H) or K562-Bw4 transfectants (I). Data were pooled from at least 2 independent experiments. Bars represent the mean on relevant graphs. $n=7-14$ mice per group. ${ }^{*} P<0.05,{ }^{*} P<0.01$, and ${ }^{* * *} P<0.001$, by multiple paired Student's $t$ tests.

huNSG mice, we also studied the inherent inhibitory potential of specific KIRs. To do so, we tested degranulation toward K562 cells stably expressing individual HLA-C1, -C2, or -Bw4 molecules (Figure 5, C, F, and I). Both educated and noneducated NK cells that carry a single KIR specific to the transfected HLA on the target cell showed reduced levels of degranulation compared with unaltered or noncognate HLA-transfected K562 cells (Figure 5, A, $\mathrm{D}$, and $\mathrm{G}$; and data not shown). Thus, despite the loss of education in NK cells from mixed reconstituted mice (Figure 5, B, E, and H), noneducated single $\mathrm{KIR}^{+} \mathrm{NK}$ cells still dampened their response and exhibited self-inhibition, as previously observed for NK cells from healthy volunteers (40). In addition to a reduced education, the inhibition of NK cell reactivity by KIR ligand-expressing targets probably contributes to NK cell tolerance in mixed chimeras.

Mixed reconstituted huNSG mice have superior immune control of EBV infection. In order to assess the functional consequence of reduced NK cell education, we evaluated immune control of EBV infection. It had been previously shown that noneducated NK cells are mainly responsible for the innate immune response to mouse CMV (MCMV) infection (41). Therefore, we investigated whether an increased abundance of noneducated NK cells would improve immune control of EBV infection. To follow the disease progression of EBV-infected huNSG mice, viral loads were assessed on a weekly basis in blood and spleen at the experimental endpoints. Viral titers were significantly reduced in both the blood and spleen of mixed reconstituted animals at the endpoints of the experiments when compared with EBV infection of single-donor reconstituted animals (Figures 6, A-D). Furthermore, we also observed a reduced $\mathrm{CD}^{+} \mathrm{T}$ cell expansion in response to $\mathrm{EBV}$ infection in mixed reconstituted huNSG mice (Figure 6E), which indicates a lower viral burden and antigen amount. However, the decreased expansion to $\mathrm{CD} 8^{+} \mathrm{T}$ cells in mixed reconstituted animals did not result in an altered $\mathrm{CD}^{+} \mathrm{T}$ cell composition (Supplemental Figure $5, \mathrm{~A}-\mathrm{H})$, nor were there differences in the $\mathrm{CD} 8^{+} \mathrm{T}$ cell effector 

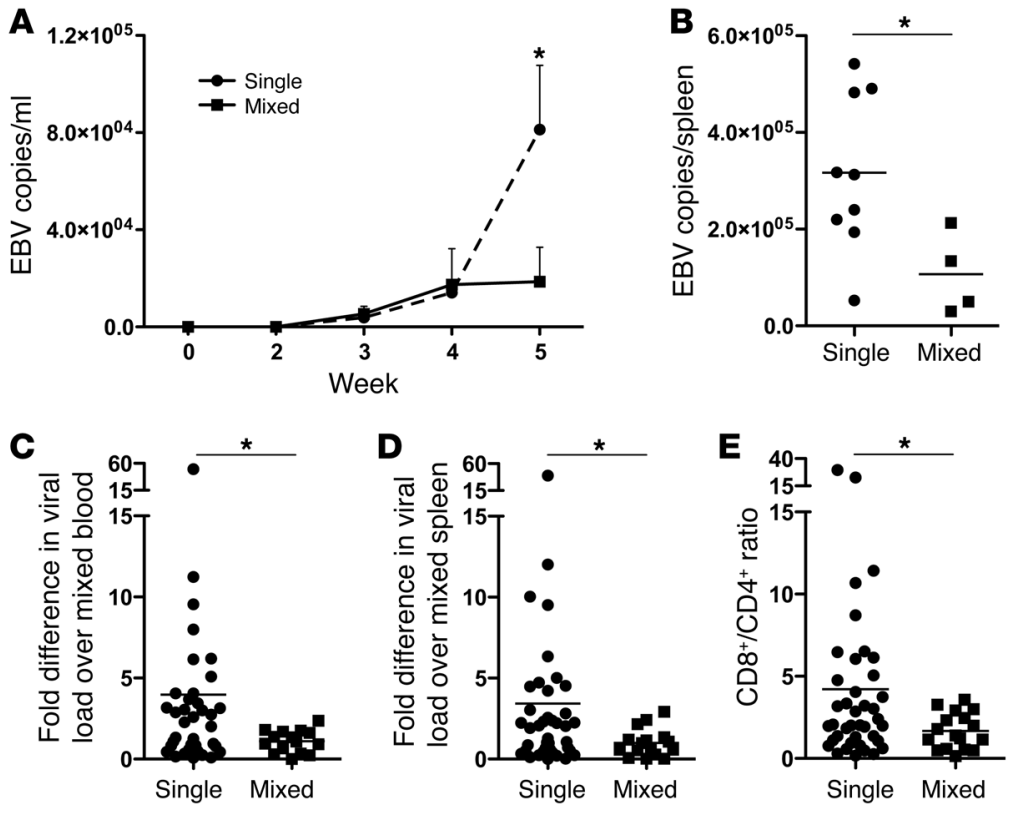

Figure 6. Improved EBV-specific immune control in mixed reconstituted huNSG mice. Viral loads in spleens of huNSC mice were read out over time in blood and at the endpoint (week 4 or 5). Homozygous single reconstituted huNSC mice (Single) were compared with mixed reconstituted mice (Mixed). (A and B) Viral loads in blood at weekly time points (A) and in spleen (B) of single versus mixed reconstituted huNSG mice for a representative experiment ( $n=3-9$ mice) group for $\boldsymbol{A}$. Data represent the mean $\pm \mathrm{SEM},{ }^{*} P<0.05$ by ANOVA with Bonferroni's correction. (C and D) Fold difference in viral load for blood (C) and spleen (D) at the endpoint normalized to the mean of mixed reconstituted group. (E) $C D 8^{+} /$ $\mathrm{CD}^{+} \mathrm{T}$ cell ratio in blood at endpoints in single versus mixed reconstituted huNSG mice. Data in $\mathbf{C}-\mathbf{E}$ were pooled from at least 3 independent experiments. $n=15-40$. Bars represent the mean. ${ }^{*} P<0.05$, by Mann-Whitney $U$ or unpaired Student's $t$ test where appropriate. memory response of the various groups (Supplemental Figure 5I). Thus, side-by-side reconstitution of huNSG mice with 2 donors, which leads to less-educated NK cells, allows for improved immune control against EBV infection.

NK cells mediate improved immune control of EBV infection in mixed reconstituted mice. To elucidate a possible role of NK cells in the improved immune control of EBV infection, huNSG mice were depleted of NK cells prior to infection with EBV (Supplemental Figure 6). Interestingly, depletion of NK cells leveled the differences previously observed in viral loads between mixed reconstituted and single reconstituted huNSG mice (Figure 7A). Moreover, viral titers in both blood and spleen of mixed reconstituted animals depleted of NK cells were significantly higher compared with viral loads in mixed reconstituted animals without depletion of NK cells (Figures 7, B and C). Additionally, the loss of NK cell-mediated protection correlated with an elevated $\mathrm{CD} 8^{+} \mathrm{T}$ cell expansion in NK cell-depleted mixed reconstituted huNSG mice (Figure 7D). Finally, in order to investigate the formation of an adaptive NK cell compartment upon EBV infection, we assessed surface markers that had been implicated in NK cell memory formation during viral infections (42-44). In accordance with published data, adaptive NK cells were phenotypically characterized by a lack of Syk expression (Supplemental Figure 7, A and B) as well as lower levels of FceRI $\gamma$ and NKp46 (Supplemental Figure 7, C and D). Interestingly, only single reconstituted huNSG mice displayed higher frequencies of adaptive NK cells, while mixed reconstituted huNSG mice appeared to have more conventional NK cells after EBV infection (Supplemental Figure 7B). Furthermore, unlike Liu et al. (44), we did not observe increased frequencies of CD2 ${ }^{+}$cells in the adaptive NK cell subset (Supplemental Figure 7E) but did find greater frequencies of NK cells carrying educated KIRs (Supplemental Figure 7F). Additionally, in a functional readout using plate-bound CD16 to stimulate NK cells, both the conventional and adaptive compartments were shown to degranulate slightly more upon EBV infection (Supplemental Figure 7G and Supplemental Figure $7 \mathrm{H}$ ). These data indicate that noneducated NK cells might play a key role in the improved control of EBV infection in mixed reconstituted huNSG mice; however, the phenotypically identifiable adaptive NK cell compartment does not appear to correlate with protection.

\section{Discussion}

Using mixed HPC reconstitution in NSG mice, we were able to assess the influence of cognate HLA absence in trans on KIR repertoire generation and NK cell education in the reconstituting human immune system. We observed that similar repertoires of $\mathrm{KIR}^{+} \mathrm{NK}$ cells reconstituted in single donor and mixed KIR ligandmismatched donor reconstituted NSG mice as well as in the fetal liver of the HPC donors used for huNSG generation. In contrast, KIR-mediated NK cell education was compromised if even a small subset of leukocytes that lacked the respective KIR ligand reconstituted in the same NSG mouse in parallel. This indicates that KIR-mediated education seems to be even more sensitive than Ly49-mediated education, with disarming taking place at a KIR ligand-negative frequency below $10 \%$ compared with $20 \%$ for the required Ly49 ligand-negative frequency in the murine system $(45,46)$. Therefore, our findings suggest that such small KIR ligand-negative leukocyte populations are able to disarm NK cells for decreased missing-self recognition of tumor cells. However, our data suggest that the availability of a low-affinity binding ligand, as is the case for the KIR2DL2/3 ligand HLA-C2 (47), can be sufficient to prevent the disarming of NK cells in trans (Figures $4 \mathrm{~B}$ and Figure 5, D and E). Hence the mechanism of disarming and which leukocyte populations are mainly involved require further investigation. Nevertheless, disarming, while disabling HLA $^{-}$tumor cell recognition, improves NK cell-mediated immune control of herpesvirus infections, as previously shown for the $\beta$-herpesvirus MCMV (41) and shown here for the $\gamma$-herpesvirus EBV (Figures 6 and 7).

In contrast to the lack of imprint of EBV infection in the NK cell repertoire, as was observed in this study, HCMV has been described to expand terminally differentiated $\mathrm{NKG}_{2} \mathrm{C}^{+} \mathrm{NK}$ cells 

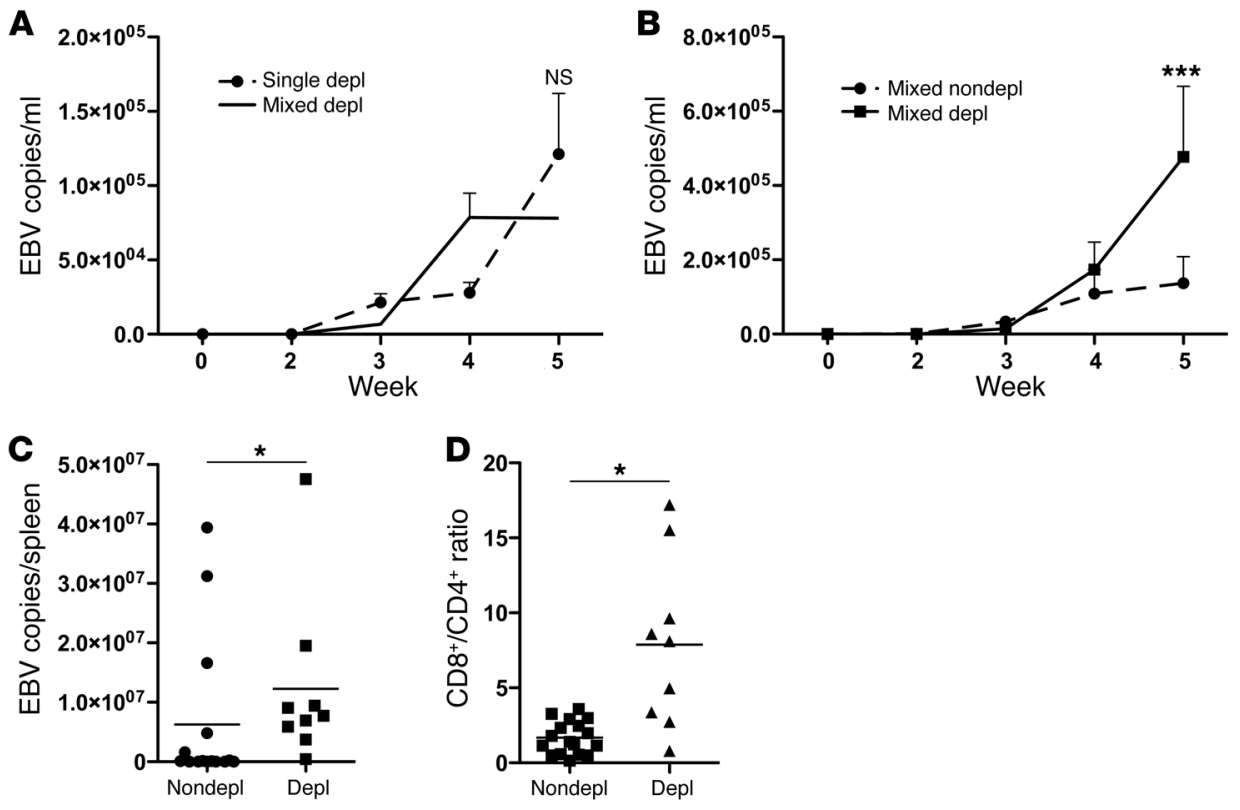

Figure 7. NK cells mediate protection in mixed reconstituted huNSG mice. Viral loads of huNSG mice were read out over time in blood and at the endpoint (week 4 or 5) in spleens. Homozygous single reconstituted huNSG were compared with mixed reconstituted animals, and non-NK cell-depleted (Nondepl) were compared with NK cell-depleted (Depl) mixed reconstituted huNSG mice. (A) Representative experiment for viral loads in blood at weekly time points in NK cell-depleted single versus NK cell-depleted mixed reconstituted huNSG mice ( $n=3-8$ mice/group). (B) Viral loads in blood over time in nondepleted (Nondepl) versus NK cell-depleted mixed reconstituted huNSG mice. (C) Endpoint viral loads in spleen for nondepleted versus NK cell-depleted (Depl) mixed reconstituted huNSG mice. (D) $C D 8^{+} / C D 4^{+} T$ cell ratio in blood at the endpoint in nondepleted versus NK cell-depleted mixed huNSG mice. Data in B-D were pooled from at least 2 independent experiments. $n=8-15$ mice per group. Data represent the mean \pm SEM or bars represent the mean. ${ }^{*} P<$ 0.05 and ${ }^{* *} P<0.001$, by ANOVA with Bonferroni's correction, Mann-Whitney $U$ test, or unpaired Student's $t$ test where appropriate.

that preferentially carry KIRs for self-HLA molecules (18). This self-HLA reactive KIR expression might be required to safeguard against immunopathology by this expanded, highly cytotoxic NK cell population. It has been suggested that this expansion might result from the engagement of the CD94/NKG2C receptor by HLA-E on HCMV-infected cells in combination with IL-15 and/ or IL-12 produced by infected or bystander myeloid cells $(48,49)$. Indeed, these cytokines might be crucial for the accumulation of terminally differentiated NK cells in HCMV carriers, because NKG2C-deficient individuals also accumulate NK cell populations, which are reminiscent in their phenotype and function of NKG2C ${ }^{+} \mathrm{NK}$ cells in the absence of this receptor (44). In contrast, EBV drives the expansion of preferentially KIR', early differentiated NK cell populations, possibly through the direct recognition of lytically EBV replicating B cells $(25,26)$ that do not efficiently produce the NK cell-differentiating cytokines IL-15 and IL-12. This does not seem to result in skewing of the NK cell repertoire. Thus, it is possibly the capacity of the target cells of these 2 herpesviruses to produce NK cell-differentiating cytokines, namely IL-12 and IL-15, that is responsible for the accumulation of terminally differentiated NK cells during HCMV and early differentiated NK cells during EBV infection, resulting, only for HCMV, in alterations of the NK cell repertoire.

This study addresses for the first time to our knowledge KIR-mediated NK cell education by cognate and noncognate HLA molecules on neighboring leukocytes, because this family of inhibitory NK cell receptors is absent in mice (4), and during transplantation with double cord blood units, usually 1 donor eliminates the other because of alloreactive responses by cotransferred $\mathrm{T}$ cells (31). Therefore, KIR-mediated NK cell education cannot be studied in these settings. Nevertheless, understanding KIR-mediated NK cell education is of clinical importance, because it has been shown that haploidentical hematopoietic cell transplantation, harnessing KIRHLA mismatch recognition, provides therapeutic benefits in adult acute myeloid leukemia (AML) and pediatric acute lymphoblastic leukemia (ALL) patients (50-52). It is thought that NK cells reconstituting from the KIR ligand-mismatched graft mediate a graft-versus-leukemia (GvL) effect via missing-self recognition of the leukemic blasts. Indeed, it has been observed that the reconstituting NK cells mediate allorecognition against the recipient's leukemic blasts to an extent similar to that of the donor's mature NK cells and that the degree of donor allorecognition of recipient cells is the best predictor for clinical efficacy $(47,53)$. These findings suggest that the donor's reconstituting human immune system components are sufficient to educate NK cells for KIR ligand-mismatched leukemia recognition, that the allogeneic residual leukemia blasts of the recipient are not able to sufficiently disarm the reconstituting NK cells, and that also the recipient's somatic cells are not able to sufficiently induce tolerance in the reconstituting donor NK cells. However, our data suggest that the GvL effect cannot be further potentiated by combining donors with high antileukemic NK cell reactivity, because their reconstituting leukocytes might disarm each other and result in diminished GvL reactivity, as observed in our study in the recognition of the human erythroleukemia cell line K562.

In contrast to possibly diminished GvL activity in mixed reconstitutions, the resulting mixed NK cell compartments might be superior in preventing EBV-associated lymphomas during transplantation. Indeed, most post-transplantation lymphoproliferative diseases (PTLDs) are $\mathrm{EBV}^{+}(23)$, and plasma EBV DNA copy numbers seem to be a reliable indicator of PTLD occurrence and treatment response $(54,55)$. This might in part reflect uncontrolled lytic EBV replication in transplant patients prior to PTLD, infecting and transforming reconstituting B cells to result in poly- or oligoclonal lymphoproliferations. This lytic replication is indeed susceptible to NK cell-mediated immune control, and non-KIR educated NK cells have been shown to preferentially target lytically EBV replicating B cells $(25,26)$. Furthermore, in the 
presence of side-by-side KIR ligand-mismatched donor reconstitution, mismatched EBV-transformed B cells might be more susceptible to NK cell lysis, while the response to their matched counterpart would still be dampened by KIR inhibition (40). Therefore, mixed HPC reconstitutions could be beneficial for controlling herpesvirus infections, resulting in mixed, less-educated NK cell compartments that more efficiently control EBV infection, as shown in this study, and that might also possibly target CMV infection in humans.

Our study, therefore, contributes to an increasing body of literature that suggests that hematopoietic cell transplantation should be tailored to address the different therapeutic needs of patients. Completely HLA-matched transplantation reduces the risk for graft-versus-host disease, but haploidentical HLA-mismatched transplantation of $\mathrm{T}$ cell-depleted grafts mediates a potent $\mathrm{GvL}$ effect by NK cell alloreactivity. In addition, mixed transplantation of $\mathrm{T}$ cell-depleted grafts might alter NK cell education for more efficient herpesvirus-specific immune control. In support of this notion, our data suggest that even minor leukocyte populations lacking cognate HLA molecules for KIRs might reduce education of the respective KIR-carrying NK cell populations for improved EBV-specific immune control.

\section{Methods}

Mice. NOD/LtSz-scid IL2R $\gamma^{\text {null }}$ (NOD-SCID $\gamma_{\mathrm{c}}{ }^{-/-}$or NSG) mice were obtained from The Jackson Laboratory and bred and maintained at the Institute of Experimental Immunology of the University of Zürich under specific pathogen-free conditions. Newborn NSG mice (1-5 days old) were irradiated with 1 Gy using a Cs or x-ray source. Five to six hours after irradiation, mice were injected intrahepatically with $1 \times$ $10^{5}$ to $2 \times 10^{5} \mathrm{CD}_{3} 4^{+}$human HPCs derived from HFL tissue obtained from Advanced Bioscience Resources. Injected HPCs were either from a single donor or were a mix from 2 donors at a 1:1 ratio, amounting to the same total cell numbers. Preparation of HFL tissue and isolation of human $\mathrm{CD} 34^{+}$cells were performed as described previously $(33,34,56)$. Reconstitution of human immune system components in mice (huNSG) was analyzed 10-12 weeks after engraftment and again just before the start of the experiments. Mice with more than $40 \%$ reconstitution of huCD $45^{+}$human immune system components in the lymphocytes of peripheral blood were used in the described experiments (average $70 \%$ huCD $45^{+}$cells of peripheral blood lymphocytes, $25 \% \mathrm{CD}^{+} \mathrm{T}$ cells of human lymphocytes, $60 \% \mathrm{CD} 19^{+} \mathrm{B}$ cells of human lymphocytes, $5 \%$ NK cells of human lymphocytes, and $80 \%$ $\mathrm{CD}^{+}$and $20 \% \mathrm{CD}^{+} \mathrm{T}$ cells of human T cells), were sex matched, and were 12-18 weeks old at the start of the experiments.

HLA typing. To perform HLA genomic typing, DNA was extracted using the QIAGEN GmbH reagent, according to the manufacturer's instructions. HLA alleles were identified by a PCR sequence-specific oligonucleotide reverse assay using commercial HLA kits (Fujirebio Diagnostics Inc.). Data analysis was performed using LIPA Interpretation Software (Fujirebio Diagnostics Inc.).

Viral infection and NK cell depletion. GFP-EBV B95-8 WT was produced in 293 cells. Titration of viral concentrates was done on Raji cells in serial dilutions and calculated as Raji-infecting units (RIU) using flow cytometric analysis of $\mathrm{GFP}^{+}$Raji cells 2 days after infection of cells. HuNSG mice were infected via i.p. injection of $1 \times 10^{5} \mathrm{RIU}$ and followed for 5 weeks. Depletion of NK cells in huNSG mice was done via i.p. injection of purified anti-NKp46 Ab (clone BAB281 provided by E. Marcenaro and A. Moretta.) in PBS on 3 consecutive days (total of $300 \mu \mathrm{g} / \mathrm{mouse}$ ). On the following day, animals were infected by inoculation via i.p. injection of $1 \times 10^{5}$ RIU EBV.

Flow cytometry. All fluorescently labeled Abs were purchased from BD Biosciences, Beckman Coulter, BioLegend, Invitrogen (Thermo Fisher Scientific), and R\&D Systems (Supplemental Table 1). Spleens were mechanically disrupted and filtered through a $70-\mu \mathrm{m}$ cell strainer before separation of mononuclear cells on Ficoll-Paque gradients. Livers were first digested in a buffer containing DNAse and Collagenase D (Roche). After being mechanically disrupted, mononuclear cells were separated using a Percoll gradient. Lysis of erythrocytes in whole blood was done with $\mathrm{NH}_{4} \mathrm{Cl}$. For intracellular staining, the Cytofix/ Cytoperm Kit from BD Biosciences was used. Cell suspensions were stained with Abs for 30 minutes on ice, washed, and analyzed on a FACSCanto or LSR Fortessa cytometer (BD Biosciences). Analysis of flow cytometric data was performed with FlowJo software (Tree Star).

K562 cells and their HLA class I transfection. K562 cells were maintained in RPMI 1640 medium (Gibco, Thermo Fisher Scientific) supplemented with gentamicin $(20 \mu \mathrm{g} / \mathrm{ml})$ and $10 \%$ FCS (Gibco, Thermo Fisher Scientific). Cell cultures were kept in a water-saturated atmosphere with $5 \% \mathrm{CO}_{2}$ at $37^{\circ} \mathrm{C}$. For the generation of HLA-Bw4-, HLA-C1-, and HLA-C2-expressing K562 cells, K562 cells were transfected with $\mathrm{HLA}^{*}{ }^{*} 51: 01, \mathrm{HLA} \mathrm{C}^{*} 03: 03$, or HLA-C ${ }^{*}$ 05:02 cDNA, respectively, inserted into the expression vector pcDNA3.1 (Invitrogen, Groningen). Briefly, K562 cells were transfected by electroporation with plasmid DNA $\left(10 \mu \mathrm{g} / 1 \times 10^{6}\right.$ cells $)$ in Opti-MEM I with Glutamax (Gibco, Thermo Fisher Scientific). Electroporation conditions were $250 \mathrm{~V}, 300 \mu \mathrm{F}$ using the Gene Pulser II (Bio-Rad). Transfected cells were selected by the addition of G418 $(1 \mathrm{mg} / \mathrm{ml})$ to the culture medium and subsequently cloned by single-cell sorting of $\mathrm{HLA}^{+}$cells using flow cytometry.

Degranulation assay. For the degranulation assay, splenic cell suspensions and nontransfected K562 or HLA-transfected K562 cells were cocultured at a ratio of 10:1 for 5 hours with addition of monensin after 1 hour, and NK cells were then analyzed for expression of the degranulation marker CD107a. Single $\mathrm{KIR}^{+} \mathrm{NK}$ cells were not pregated on NKG2A. Degranulation is presented as the difference between degranulation after K562 coculture and spontaneous degranulation ( $\triangle \% \mathrm{CD} 107 \mathrm{a})$.

CD16 stimulation assay. For the degranulation assay, NUNC Multisorp plates were coated with $5 \mu \mathrm{g} / \mathrm{ml}$ anti-CD16 (clone 3G8, BioLegend) overnight at $4^{\circ} \mathrm{C}$. Thawed splenic cell suspensions were rested overnight in RPMI 1640 medium (Gibco BRL) supplemented with gentamicin $(20 \mu \mathrm{g} / \mathrm{ml})$ and $10 \%$ FCS (Gibco BRL) before being transferred onto coated plates for 6 hours with addition of monensin and brefeldin A after 1 hour. NK cells were then analyzed by flow cytometry for expression of the degranulation marker CD107a. Degranulation is presented as the difference between degranulation after CD16 cross-linking and spontaneous degranulation ( $\triangle \% \mathrm{CD} 107 \mathrm{a})$.

Quantification of EBV load by real-time PCR. Total DNA from whole blood was extracted using the NucliSENS EasyMAG System (bioMérieux) according to the manufacturer's instructions. Splenic tissue was processed using a QIAamp DNA Tissue Kit (QIAGEN) according to the manufacturer's protocol. Quantitative analysis of EBV DNA was performed by the TaqMan (Applied Biosystems) realtime PCR technique as previously described (57), with modified prim- 
ers for the Bam $\mathrm{H} 1 \mathrm{~W}$ fragment (5'-CTTCTCAGTCCAGCGCGTTT-3' and $5^{\prime}$-CAGTGGTCCCCCTCCCTAGA-3') and a fluorogenic probe (5'-FAM-CGTAAGCCAGACAGCAGCCAATTGTCAG-TAMRA-3'). All PCRs were run on an ABI Prism 7700 Sequence Detector (Applied Biosystems), and samples were analyzed in duplicate. No EBV DNA was detected in the blood of mock-infected animals for the duration of the experiment. Mice were considered uninfected if EBV DNA was not detected in the blood or spleen during the experiment.

Statistics. All data were analyzed with an unpaired or paired, 2-tailed Student's $t$ test, a Mann-Whitney $U$ test, or ANOVA with Bonferroni's correction. A $P$ value of less than 0.05 was considered statistically significant. Statistical analysis and generation of graphs were performed with GraphPad Prism, Version 5.0a (GraphPad Software).

Study approval. All animal protocols were approved by the cantonal veterinary office of the canton of Zürich, Switzerland (protocols 148/2011 and 209/2014). All studies involving human samples were reviewed and approved by the cantonal ethics committee of Zürich, Switzerland (protocol KEK-StV-Nr.19/08).

\section{Author contributions}

VL and AR conducted the experiments. GP and GF performed the HLA typing. VB and KJM were instrumental in performing the KIR repertoire analysis. EM and AM provided essential reagents for the study. CG generated the K562 transfectants. RC and AZ determined the viral titers. VL, OC, and CM designed the experiments, analyzed the data, and wrote the manuscript.

\section{Acknowledgments}

We thank W. Held for critically reading the manuscript. This work was supported by grants from Cancer Research Switzerland (KFS3234-08-2013); Worldwide Cancer Research (14-1033); SPARKS (15UOZ01), KFSP ${ }^{\mathrm{MS}}$, and KFSPHHLD of the University of Zürich; the Sobek Foundation; the Swiss Vaccine Research Institute and the Swiss National Science Foundation (310030_162560 and CRSII3_160708, to CM). OC is the recipient of a grant from Cancer Research Zürich.

Address correspondence to: Christian Münz, Viral Immunobiology, Institute of Experimental Immunology, University of Zürich, Winterthurerstrasse 190, 8057 Zürich, Switzerland. Phone: 41.44.635.3716; E-mail: christian.muenz@uzh.ch. Or to: Obinna Chijioke, Institute of Surgical Pathology, University Hospital Zürich, Schmelzbergstrasse 12, 8091 Zürich, Switzerland. Phone: 41.44.255.2716; E-mail: obinna.chijioke@usz.ch.
1. Herberman RB, Nunn ME, Lavrin DH. Natural cytotoxic reactivity of mouse lymphoid cells against syngeneic acid allogeneic tumors. I. Distribution of reactivity and specificity. Int $J$ Cancer. 1975;16(2):216-229.

2. Trinchieri G, Santoli D. Anti-viral activity induced by culturing lymphocytes with tumor-derived or virus-transformed cells. Enhancement of human natural killer cell activity by interferon and antagonistic inhibition of susceptibility of target cells to lysis. JExp Med. 1978;147(5):1314-1333.

3. Kiessling R, Klein E, Wigzell H. "Natural” killer cells in the mouse. I. Cytotoxic cells with specificity for mouse Moloney leukemia cells. Specificity and distribution according to genotype. Eur J Immunol. 1975;5(2):112-117.

4. Long EO, Kim HS, Liu D, Peterson ME, Rajagopalan S. Controlling natural killer cell responses: integration of signals for activation and inhibition. Annu Rev Immunol. 2013;31:227-258.

5. Martinet L, Smyth MJ. Balancing natural killer cell activation through paired receptors. Nat Rev Immunol. 2015;15(4):243-254.

6. Moretta A, et al. Activating receptors and coreceptors involved in human natural killer cell-mediated cytolysis. Annu Rev Immunol. 2001;19:197-223.

7. Pradeu T, Jaeger S, Vivier E. The speed of change: towards a discontinuity theory of immunity? Nat Rev Immunol. 2013;13(10):764-769.

8. Elliott JM, Yokoyama WM. Unifying concepts of MHC-dependent natural killer cell education. Trends Immunol. 2011;32(8):364-372.

9. Goodridge JP, Önfelt B, Malmberg KJ. Newtonian cell interactions shape natural killer cell education. Immunol Rev. 2015;267(1):197-213.

10. Chalifour A, et al. A Role for cis Interaction between the Inhibitory Ly49A receptor and MHC class I for natural killer cell education. Immunity. 2009;30(3):337-347.
11. Ebihara T, Jonsson AH, Yokoyama WM. Natural killer cell licensing in mice with inducible expression of MHC class I. Proc Natl Acad Sci US A. 2013;110(45):E4232-E4237.

12. Bessoles S, Angelov GS, Back J, Leclercq G, Vivier E, Held W. Education of murine NK cells requires both cis and trans recognition of MHC class I molecules. J Immunol. 2013;191(10):5044-5051.

13. Joncker NT, Shifrin N, Delebecque F, Raulet DH. Mature natural killer cells reset their responsiveness when exposed to an altered MHC environment. J Exp Med. 2010;207(10):2065-2072.

14. Haas P, et al. NK-cell education is shaped by donor HLA genotype after unrelated allogeneic hematopoietic stem cell transplantation. Blood. 2011;117(3):1021-1029.

15. Ruggeri L, et al. Role of natural killer cell alloreactivity in HLA-mismatched hematopoietic stem cell transplantation. Blood.1999;94(1):333-339.

16. Viant C, Fenis A, Chicanne G, Payrastre B, Ugolini S, Vivier E. SHP-1-mediated inhibitory signals promote responsiveness and anti-tumour functions of natural killer cells. Nat Commun. 2014;5:5108.

17. Tripathy SK, et al. Continuous engagement of a self-specific activation receptor induces NK cell tolerance. JExp Med. 2008;205(8):1829-1841.

18. Béziat V, et al. NK cell responses to cytomegalovirus infection lead to stable imprints in the human KIR repertoire and involve activating KIRs. Blood. 2013;121(14):2678-2688.

19. Orange JS. Natural killer cell deficiency. J Allergy Clin Immunol. 2013;132(3):515-525; quiz 526.

20. Taylor GS, Long HM, Brooks JM, Rickinson AB, Hislop AD. The immunology of Epstein-Barr virus-induced disease. Annu Rev Immunol. 2015;33:787-821.

21. Balfour $\mathrm{HH}$, et al. Behavioral, virologic, and immunologic factors associated with acquisition and severity of primary Epstein-Barr virus infection in university students. J Infect Dis.
2013;207(1):80-88

22. Luzuriaga K, Sullivan JL. Infectious mononucleosis. N Engl J Med. 2010;362(21):1993-2000.

23. Cesarman E. Gammaherpesviruses and lymphoproliferative disorders. Annu Rev Pathol. 2014;9:349-372.

24. Williams H, et al. The immune response to primary EBV infection: a role for natural killer cells. Br J Haematol. 2005;129(2):266-274.

25. Azzi T, et al. Role for early-differentiated natural killer cells in infectious mononucleosis. Blood. 2014;124(16):2533-2543.

26. Chijioke O, et al. Human natural killer cells prevent infectious mononucleosis features by targeting lytic Epstein-Barr virus infection. Cell Rep. 2013;5(6):1489-1498.

27. Dunmire SK, Grimm JM, Schmeling DO, Balfour $\mathrm{HH}, \mathrm{Hogquist}$ KA. The incubation period of primary Epstein-Barr virus infection: viral dynamics and immunologic events. PLoS Pathog. 2015;11(12):e1005286.

28. Pappworth IY, Wang EC, Rowe M. The switch from latent to productive infection in epsteinbarr virus-infected $\mathrm{B}$ cells is associated with sensitization to NK cell killing. J Virol. 2007;81(2):474-482.

29. Barker JN, et al. Transplantation of 2 partially HLA-matched umbilical cord blood units to enhance engraftment in adults with hematologic malignancy. Blood. 2005;105(3):1343-1347.

30. Scaradavou A, et al. Double unit grafts successfully extend the application of umbilical cord blood transplantation in adults with acute leukemia. Blood. 2013;121(5):752-758.

31. Gutman JA, et al. Single-unit dominance after double-unit umbilical cord blood transplantation coincides with a specific CD8+ T-cell response against the nonengrafted unit. Blood. 2010;115(4):757-765.

32. Hashem H, Lazarus HM. Double umbilical cord 
blood transplantation: relevance of persistent mixed-unit chimerism. Biol Blood Marrow Transplant. 2015;21(4):612-619.

33. Strowig T, et al. Human NK cells of mice with reconstituted human immune system components require preactivation to acquire functional competence. Blood. 2010;116(20):4158-4167.

34. Strowig T, et al. Priming of protective T cell responses against virus-induced tumors in mice with human immune system components. J Exp Med. 2009;206(6):1423-1434.

35. Gumá M, Angulo A, Vilches C, Gómez-Lozano N, Malats N, López-Botet M. Imprint of human cytomegalovirus infection on the NK cell receptor repertoire. Blood. 2004;104(12):3664-3671.

36. Charoudeh HN, et al. Quantity of HLA-C surface expression and licensing of KIR2DL+ natural killer cells. Immunogenetics. 2012;64(10):739-745.

37. Anfossi N, et al. Human NK cell education by inhibitory receptors for MHC class I. Immunity. 2006;25(2):331-342.

38. Burt BM, et al. The lytic potential of human liver NK cells is restricted by their limited expression of inhibitory killer Ig-like receptors. J Immunol. 2009;183(3):1789-1796.

39. Stegmann KA, et al. CXCR6 marks a novel subset of T-bet(lo)Eomes(hi) natural killer cells residing in human liver. Sci Rep. 2016;6:26157.

40. Yu J, Heller G, Chewning J, Kim S, Yokoyama WM, Hsu KC. Hierarchy of the human natural killer cell response is determined by class and quantity of inhibitory receptors for self-HLA-B and HLA-C ligands. J Immunol. 2007;179(9):5977-5989.

41. Orr MT, Murphy WJ, Lanier LL. 'Unlicensed' natural killer cells dominate the response to cytomegalovirus infection. Nat Immunol.
2010;11(4):321-327.

42. Schlums H, et al. Cytomegalovirus infection drives adaptive epigenetic diversification of NK cells with altered signaling and effector function. Immunity. 2015;42(3):443-456.

43. Lee J, et al. Epigenetic modification and antibody-dependent expansion of memory-like NK cells in human cytomegalovirus-infected individuals. Immunity. 2015;42(3):431-442.

44. Liu LL, et al. Critical role of CD2 co-stimulation in adaptive natural killer cell responses revealed in NKG2C-deficient humans. Cell Rep. 2016;15(5):1088-1099.

45. Johansson MH, Bieberich C, Jay G, Kärre K, Höglund P. Natural killer cell tolerance in mice with mosaic expression of major histocompatibility complex class I transgene. J Exp Med. 1997;186(3):353-364.

46. Johansson MH, Höglund P. Low number of $\mathrm{H}-2 \mathrm{Dd}$-negative haematopoietic cells in mixed bone marrow chimeras convey in vivo tolerance to H-2Dd-negative cells but fail to prevent resistance to H-2Dd-negative leukaemia. Scand J Immunol. 2004;59(1):71-78.

47. Pende D, et al. Anti-leukemia activity of alloreactive NK cells in KIR ligand-mismatched haploidentical HSCT for pediatric patients: evaluation of the functional role of activating KIR and redefinition of inhibitory KIR specificity. Blood. 2009;113(13):3119-3129.

48. Gumá M, et al. Expansion of CD94/ $\mathrm{NKG} 2 \mathrm{C}+\mathrm{NK}$ cells in response to human cytomegalovirus-infected fibroblasts. Blood. 2006;107(9):3624-3631.

49. Rölle A, et al. IL-12-producing monocytes and HLA-E control HCMV-driven NKG2C+ NK cell expansion. J Clin Invest. 2014;124(12):5305-5316.

50. Velardi A, Ruggeri L, Mancusi A. Killer-cell immunoglobulin-like receptors reactivity and outcome of stem cell transplant. Curr Opin Hematol. 2012;19(4):319-323.

51. Ruggeri L, et al. Effectiveness of donor natural killer cell alloreactivity in mismatched hematopoietic transplants. Science. 2002;295(5562):2097-2100.

52. Locatelli F, et al. Outcome of children with highrisk acute myeloid leukemia given autologous or allogeneic hematopoietic cell transplantation in the aieop AML-2002/01 study. Bone Marrow Transplant. 2015;50(2):181-188.

53. Pietra G, et al. Human natural killer cells: news in the therapy of solid tumors and high-risk leukemias. Cancer Immunol Immunother. 2016;65(4):465-476.

54. Tsai DE, et al. EBV PCR in the diagnosis and monitoring of posttransplant lymphoproliferative disorder: results of a two-arm prospective trial. Am J Transplant. 2008;8(5):1016-1024.

55. Ruf S, et al. Comparison of six different specimen types for Epstein-Barr viral load quantification in peripheral blood of pediatric patients after heart transplantation or after allogeneic hematopoietic stem cell transplantation. J Clin Virol. 2012;53(3):186-194.

56. White RE, et al. EBNA3B-deficient EBV promotes B cell lymphomagenesis in humanized mice and is found in human tumors. JClin Invest. 2012;122(4):1487-1502.

57. Berger C, Day P, Meier G, Zingg W, Bossart W, Nadal D. Dynamics of Epstein-Barr virus DNA levels in serum during EBV-associated disease. JMed Virol. 2001;64(4):505-512. 